\title{
CARACTERIZACION Y ANALISIS DE LA VARIABILIDAD GENÉTICA DE LA GRANADILLA (Passiflora ligularis Juss.) EN COLOMBIA EMPLEANDO MARCADORES MICROSATÉLITES ${ }^{1}$
}

\author{
NATHALIA BERNAL-PARRA², JOHN OCAMPO-PÉREZ ${ }^{3}$, \\ JAVIER HERNÁNDEZ-FERNÁNDEZ ${ }^{4}$
}

RESUMEN-La granadilla es la segunda especie en importancia económica del género Passiflora y Colombia es el principal productor del mundo con 53.000 t/año. Son pocos los estudios sobre la diversidad intraespecifica en la especie que permitan establecer las relaciones genéticas entre individuos. El objetivo de esta investigación fue explorar la variabilidad genética de la granadilla cultivada en Colombia por medio de marcadores microsatélites. Diez marcadores microsatélites fueron evaluados en 41 accesiones (82 individuos) provenientes de los principales departamentos productores. Un total de cinco microsatélites fueron amplificados con 66 alelos identificados y un promedio de 12,2, entre ellos 7 únicos y 13 raros. Los índices de diversidad mostraron un contenido de información polimórfica de 0,74 (PIC), y una heterocigocidad promedio observada $(\mathrm{Ho})$ y esperada $(\mathrm{He})$ de 0,98 y 0,96 bajo condiciones de equilibrio de Hardy-Weinberg. La distancia genética promedio dentro y entre poblaciones fue de 0,65 y 0,80 , siendo Boyacá, Valle del Cauca y Putumayo los más distantes $(>0,87)$. Los análisis de clasificación arbórea $(n j)$ y factorial de correspondencia múltiple (AFCM) revelaron poca estructuración geográfica de las accesiones y dispersión de los individuos de un mismo origen. La carencia de estructuración y la alta variabilidad intraespecífica podría explicarse por el fenómeno de alogamia presente en la especie y el intercambio de semillas entre productores. En conclusión, estos resultados sugieren una evaluación agromorfológica complementaria que permita establecer la variabilidad genética total e implementar un programa de mejoramiento genético por medio de la selección asistida de genotipos superiores en búsqueda de cultivares más productivos y resistentes a problemas fitosanitarios que afectan los cultivos.

Palabras clave: Passiflora, fruta, marcadores moleculares, variabilidad intraespecífica, fitomejoramiento.

\section{CHARACTERIZATION AND ANALYSIS OF THE GENETIC VARIABILITY OF SWEET PASSION FRUIT (Passiflora ligularis Juss.) IN COLOMBIA USING MICROSATELLITE MARKERS}

\begin{abstract}
Sweet passion fruit is the second economically important species of the genus Passiflora and Colombia is the largest producer in the world with 53,000 t/year. There are however, few studies on intraspecific diversity within the species that establish genetic relationships between individuals. The objective of this research was to explore the genetic variability of cultivated sweet passion fruit in Colombia using microsatellite markers. Ten microsatellite markers were evaluated in 41 accessions ( 82 individuals) from the main producing departments in the country. A total of five microsatellites were amplified with 66 alleles identified and an average of 12.2, including 7 unique and 13 rare. Diversity indexes showed a polymorphic information content of 0.74 (PIC), and an observed $(\mathrm{Ho})$ and expected $(\mathrm{He})$ heterozygosity average of 0.98 and 0.96 under Hardy-Weinberg equilibrium conditions. The average genetic distance within and among populations was 0.65 and 0.80 , being Boyacá, Valle del Cauca and Putumayo the most distant $(>0.87)$. Classification Trees $(n j)$ and Multiple Correspondence Factorial Analysis (MCA) revealed little geographical structuring of accessions and individuals dispersal of a common origin. The lack of structure and high intraspecific variability could be explained by the phenomenon of allogamy present in the species and seed exchange among farmers. In conclusion, these results suggest a complementary agromorphological assessment in order to establish the total genetic variability and implement a breeding program through assisted selection of superior genotypes in search of more productive and resistant cultivars to phytosanitary problems that affect crops.

Index terms: Passiflora, fruit, molecular markers, intraespecific variability, plant breeding.

\footnotetext{
${ }^{1}$ (Trabalho 251-13). Recebido em: 01-08-2013). Aceito para publicação em: 04-05-2014 ${ }^{2}$ Estudiante de tesis de pregrado en Biología.

${ }^{3}$ Docente investigador, Univ. Nacional de Colombia sede Palmira, Facultad de Ciencias Agropecuarias, Carrera 32 No 12-00 Chapinero, Vía Candelaria // Centro Internacional de Agricultura Tropical - CIAT-DAPA, A.A 6713, CIAT/Cali, Colombia. Email: jaocampop@unal.edu.co

${ }^{4}$ Docente investigador, Univ. Jorge Tadeo Lozano, Facultad de Ciencias Naturales e Ingeniería, Dep.de Ciencias Naturales y Ambientales, Cra. 4 No 22-61, Bogotá, D.C., Colombia. Email: javier.hernandez@utadeo.edu.co
} 


\section{INTRODUCCIÓN}

La granadilla (Passiflora ligularis Juss.) es la segunda especie en importancia económica del género Passiflora por poseer un fruto comestible y comercializado en mercados nacionales e internacionales. Esta especie es originaria de los Andes tropicales, desde Bolivia a Venezuela entre los 1.500 a 2.600 m.s.n.m. (OCAMPO et al., 2007a), e introducida a otros países como México, Costa Rica, Kenia, Australia y Nueva Zelanda. La especie es diploide $(2 n=18)$ con flores hermafroditas y una polinización prevalentemente alógama (Snow; MACDOUGAL, 1992). El tipo de polinización es favorecida por la posición de las anteras debajo del estigma y es realizada principalmente por abejorros de los géneros Xylocopa sp. y Epicharis sp. (FRANCO et al., 2007). La abundancia de los polinizadores en los cultivos de granadilla puede incrementar la productividad y la variación genética debido al constante intercambio de polen entre plantas.

El principal productor a nivel mundial de granadilla es Colombia con 4.500 has cultivadas y una producción aproximada de 55.000 t/año, concentradas en los departamentos del Huila, Cundinamarca y Boyacá. El cultivo es gran generador de empleos rurales (210 jornales/h/año) y contribuye sustancialmente a la economía campesina por su constante producción y rentabilidad. Sin embargo, un factor limitante en el desarrollo del cultivo es el gran número de plagas y enfermedades, con considerables efectos negativos sobre la producción. Además, los parámetros fitosanitarios en la producción de material de siembra por parte de los viveros colombianos no son controlados y estrictos en su mayoría. Otro problema es la falta de programas de fitomejoramiento que pueden ofrecer a los productores cultivares de mayor calidad genética y responder a los problemas adversos que afectan los cultivos de granadilla, como precocidad, rendimiento, y resistencia a plagas y enfermedades (Dasiops sp., Fusarium sp., y virus SMV).

La diversidad morfológica en formas y colores de las especies del género Passiflora es muy amplia, tanto en las silvestres como las cultivadas (Ocampo et al., 2007a). En las especies comercializadas, como la granadilla, el maracuyá ( $P$. edulis f. flavicarpa Degener) y la gulupa ( $P$. edulis f. edulis Sims) los productores llevan a cabo la selección masal o fenotípica de los mejores frutos de dos o tres plantas de alto rendimiento para establecer o renovar los nuevos cultivos en función de sus observaciones de un ideotipo impuesto por el mercado local o internacional (Ocampo et al., 2013). Esta práctica de selección, puede contribuir con la reducción de la variabilidad en los cultivos por depresión endogámica en las generaciones futuras. Sin embargo, el fenómeno de alogamia presente en la especie, la amplia distribución geográfica de los cultivos y el intercambio de semillas entre productores mantienen una considerable variabilidad genética en los materiales cultivados en Colombia. Por otro lado, el conocimiento de la diversidad genética es de capital importancia para definir el valor potencial de los recursos genéticos, que permita implementar estrategias para el aprovechamiento, la conservación y la utilización de la variabilidad intra e interespecífica de las poblaciones.

Los marcadores moleculares son una herramienta que permiten estudiar el polimorfismo o la variabilidad genética en diferentes organismos, ya que pueden acceder directamente al genoma, permitiendo caracterizar y estimar las relaciones genéticas entre grupos de interés (SAMAGN et al., 2006). Los primeros estudios sobre variabilidad en Passiflora se llevaron a cabo con el uso de marcadores RAPD (FAJARDO et al., 1998; CROCHEMORE et al., 2003) y RFLP-cpDNA (SÁNCHEZ et al., 1999) en especies de los subgéneros Passifiora, Tacsonia, Decaloba y Distephana. Sin embargo, el tamaño y composición de la muestra no permitieron una interpretación a nivel inter e intraespecífica en los individuos. En contraste, Segura et al. (2002) y Ocampo et al. (2004) reportaron una alta variabilidad genética entre y dentro de las principales especies cultivadas de Passiflora (P. edulis f. flavicarpa, P. ligularis, $P$. edulis f. edulis, $P$. tarminaiana Coppens $\&$ Barney, P. tripartita var. mollissima Holm-Nielsen $\&$ Jorgensen, $P$. quadrangularis L. , $P$. maliformis L. y $P$. alata Curtis) con el uso de marcadores AFLP, sugiriendo la existencia de una fuente de genes de interés en los genotipos evaluados.

Por otro lado, los marcadores microsatélites son considerados por diversos autores como la herramienta más poderosa para estudios de genética de poblaciones (BILLOTTE et al., 1999; KALIA et al., 2011) e identificación de individuos estrechamente emparentados (OCAMPO et al., 2007b; GONÇALVES-VIDIGAL; BENCHIMOL, 2011), ya que son muy polimórficos, presentan herencia mendeliana, son codominantes, fáciles de analizar, repetitivos y altamente automatizables. A pesar de estas ventajas, existen pocos estudios sobre la diversidad en Passiflora con este tipo de marcadores y solo en las especies $P$. edulis $\mathrm{f}$. flavicarpa (OLIVEIRA et al., 2005; SANTOS et al., 2011), P. alata (PADUA et al., 2005) y P. edulis 
f. edulis (ORTIZ et al., 2012) se han implementado. El uso de los microsatélites en la granadilla no han sido reportados, y los estudios con otros tipos de marcadores como AFLP (Ocampo et al., 2004), RAPD (AUKAR et al., 2004) y ncpGs (YOCKTENG et al., 2004) se han realizado específicamente para establecer las relaciones interespecíficas en el género Passiflora. Estos resultados no revelan con claridad una estructura genética en la granadilla, debido a que los genotipos evaluados provienen de un mismo origen geográfico y el tamaño de la muestra limita la interpretación a nivel intraespecífico.

En general, la información disponible sobre la variabilidad genética en la granadilla es incipiente $\mathrm{y}$ no permite determinar las relaciones entre y dentro de accesiones de diferentes orígenes. Además, el desconocimiento de la estructura genética en la especie dificulta establecer las bases de un programa de selección y mejoramiento para la obtención de cultivares más productivos y tolerantes a problemas fitosanitarios. Por estas razones, esta investigación tuvo como objetivo caracterizar y estudiar el nivel de variabilidad genética presente en genotipos cultivados de granadilla (P. ligularis) provenientes de 10 departamentos colombianos mediante el uso de marcadores microsatélites.

\section{MATERIALES Y MÉTODOS}

\section{Material vegetal}

Un total de 82 individuos representados por 41 accesiones provenientes de 10 departamentos fueron colectados en fincas de productores y presentados en la Tabla 1. La Colección Nacional de Granadilla está localizada en la granja Tesorito de la Universidad de Caldas a 2.280 m.s.n.m (Manizales, Caldas) y cada accesión está constituida por cinco individuos provenientes de semilla de polinización abierta de cultivos comerciales.

\section{Extracción de ADN}

El ADN se extrajo de hojas jóvenes provenientes de plantas de 24 meses de edad y empleando el método de extracción vegetal reportado por Doyle y Doyle (1991). La cuantificación se realizó por comparación visual con ADN estándar de concentración conocida (Hyperladder V Bioline, California U.S.A.), después de una electroforésis en gel de agarosa al 1\% (empleando un transiluminador UV.) y teñido con bromuro de etidio.

\section{Amplificación por PCR y electroforesis}

El estudio fue llevado acabo con 10 microsatélites polimórficos aislados y caracterizados en el maracuyá ( $P$. edulis f. flavicarpa) por Oliveira et al. (2005). Igualmente, las condiciones de amplificación vía PCR fueron las reportadas para el maracuyá por el mismo autor, con algunas modificaciones realizadas en la concentración del $\mathrm{MgCl}_{2}$, la Taq Polimerasa y las temperaturas de anillamiento. La PCR se realizó en un termociclador de bloque TC9600-G MultiGene Gradient Thermal Cycler (Edison, NJ, USA) en un volumen final de 15 $\mu$ l. La mezcla de reacción incluyó: $1 \mathrm{X}$ Tampón de PCR, $2 \mathrm{mM}$ de $\mathrm{MgCl}_{2}, 0,2 \mathrm{mM}$ de dNTP's, $0,2 \mu \mathrm{M}$ de cada uno de los oligonulceótidos-primers, una unidad de Taq ADN polimerasa y $10 \mathrm{ng}$ de ADN. El programa de la PCR incluyó una denaturación de 5 min a $93^{\circ} \mathrm{C}$, y seguidamente se realizaron 36 ciclos de $40 \mathrm{~s}$ a $94^{\circ} \mathrm{C}, 40 \mathrm{~s}$ a la temperatura de anillamiento de cada primer, entre $52-56^{\circ} \mathrm{C}$ (Tabla 2 ) y $50 \mathrm{~s}$ de extensión a $72^{\circ} \mathrm{C}$, finalizando con una extensión a $72^{\circ} \mathrm{C}$ por $5 \mathrm{~min}$. Las amplificaciones se revelaron en una electroforesis de agarosa al 2,5\% teñidos con bromuro de etidio, y los fragmentos posteriormente confirmados se revelaron en una electroforesis en geles de poliacrilamida al $8 \%$ y teñidos con nitrato de plata. Finalmente, los fragmentos revelados en los geles fueron registrados con la ayuda de una lámpara de luz blanca (Hoefer Macro Vue Vis 45) y fotografiados con una cámara digital (Nikon Coolpix S550, 10.0 Mp).

\section{Análisis de datos}

Las imágenes de los geles fueron codificadas como alelos (fragmentos) y el número y peso se determinaron con la ayuda del software Vision Works LS. La riqueza alélica, frecuencia, heterocigosidad esperada $(\mathrm{He})$ y observada $(\mathrm{Ho})$, el contenido de información polimórfica (PIC) y el test de equilibrio de Hardy-Weinberg (HW) fueron calculados y ejecutados con el programa Genetix 4.05. Las relaciones entre individuos y la distancia genética fueron estimadas con el coeficiente de Dice (1945) para cada locus. La matriz de similaridad fue empleada para el análisis factorial de correspondencia múltiple (AFCM) y la construcción del dendograma por el método del vecino más próximo neighbor joining (SAITOU y NEI, 1987) con las distancias de Sokal \& Michener y valores de bootstrap de 1.000 réplicas para evaluar la solidez del árbol. Estos análisis fueron llevados a cabo con la ayuda del programa genético DARwin 6. 


\section{RESULTADOS}

Análisis de los microsatélites SSR

El 50\% de los marcadores evaluados y reportados por Oliveira et al. (2005) en maracuyá presentaron amplificación en los 82 individuos de granadilla estudiados. Los cinco marcadores seleccionados identificaron 66 alelos, con un rango entre 11 a 16 y un promedio de 12,2 por locus (Tabla 2). Los marcadores con mayor riqueza alélica fueron AY768786 ((GTTGTG) $\left.)_{4}\right)$ y AY768785 ((AG) $\left.)_{22}\right)$ con 16 y 14 alelos, y el peso promedio de cada alelo fue de 195,5 pares de bases y con rango de 84 a 307 pb. Entre el total de alelos se identificaron 7 únicos y 13 raros (frecuencia $\leq 0,060$ ) con particular distribución en las accesiones del Valle del Cauca (ValLig02, ValLig03, ValLig04, ValLig05), Boyacá (BoyLig01, BoyLig05), Caldas (CalLig01, CalLig02, CalLig06, CalLig07), Quindío (QuiLig01, QuiLig02), Tolima (TolLig01), Cundinamarca (CunLig06) y Putumayo (PutLig01).

En cuanto al contenido de información polimórfica (PIC), se encontraron valores superiores a 0,74 en cuatro de los cinco marcadores, el valor más alto lo presentó AY768786 con 0,90. El valor general de PIC para los cuatro marcadores más polimórficos fue $0,84, y$ de acuerdo con Botstein et al. (1980) estos son altamente informativos $(\geq 0,50)$. Por otra lado, el marcador AY768790 ((GT) $\left.)_{7}\right)$ presentó el valor de PIC más bajo $(0,74)$, debido a que uno de los 11 alelos observados (151 pb) está presente en la mayoría de los individuos de la población con una frecuencia de 0,354.

Los índices de diversidad genética evidencian un alto polimorfismo observado para los microsatétiles evaluados (Tabla 1). La heterocigocidad observada ( $\mathrm{Ho}$ ) y esperada $(\mathrm{He})$ promedio fue de 0,98 y 0,96 , y en la mayoría de los marcadores $H o$ fue superior, lo que sugiere un exceso de heterocigotos observados en la población bajo condiciones de equilibrio de HardyWeinberg (HW). Por el contrario, en los marcadores AY768786 y AY768787 ((TG) $)_{8}$ T(TA) $)_{5}$ ) la Ho es igual e inferior a la $\mathrm{He}$, indicando que las frecuencias alélicas son estables de una generación a otra o que hay una tendencia a la endogamia, respectivamente.

Por otro lado, los marcadores no presentaron diferencias significativas respecto a los valores de He según las proporciones de HW. Las similitudes pueden estar asociadas al fenómeno de alogamia presente en la especie y podrían estar contribuyendo al aumento de variabilidad genética en las poblaciones evaluadas.

\section{Diversidad Genética}

El valor promedio de distancia genética (DICE, 1945) total entre y dentro de la población fue de 0,735 , con valores que oscilaron desde 0,368 hasta 0,871 (Tabla 3 ). Las menores distancias se presentaron entre individuos de un mismo departamento, como es el caso de Putumayo $(0,368)$ y Tolima (0,372). En contraste, los departamentos que mostraron mayores distancias entre sus individuos fueron el Valle del Cauca $(0,655)$ y Cundinamarca $(0,595)$, a pesar de la poca distancia entre los cultivos. A nivel inter departamento, la distancia promedio alcanzó 0,741, y los menos similares con los demás fueron las accesiones de Boyacá $(0,800)$, Valle de Cauca $(0,790)$ y Putumayo $(0,788)$. El nivel de distancia entre los individuos de cada departamento es mayor que dentro de ellos, lo que sugiere una elevada heterogeneidad y confirman la existencia de una alta variabilidad intraespecífica en la granadilla cultivada en Colombia.

Análisis de clasificación - neighbor joining Las accesiones se clasificaron por el método del vecino más próximo y con distancias euclidianas, a partir de los cinco marcadores microsatélites polimórficos seleccionados. El árbol de clasificación mostró tres grandes grupos principales mal soportados (bootstrap $<50 \%$ ), con una leve estructuración geográfica y una alta diferenciación entre los individuos de una misma accesión (Figura 1). El primer grupo reúne el mayor número de individuos y está compuesto por dos ramas principales, donde se encuentran las accesiones de Tolima (TolLig), Valle del Cauca (ValLig), Antioquia (AntLig), Huila (HuiLig) y Putumayo (PutLig) de manera dispersa. El grupo dos está representado por dos ramas bien definidas y constituidas esencialmente por la mayoría de las accesiones de Caldas (CalLig) y Cundinamarca (CunLig) y algunos individuos de los departamentos de Risaralda (RisLig) y Huila (HuiLig). El grupo tres con 14 individuos es el más pequeño y al igual que los otros aparecen dos ramas definidas, con prevalencia de las accesiones de Boyacá (BoyLig) y Quindío (QuiLig). Aunque muchas de las accesiones que se encuentran relativamente asociadas dentro de los grupos, los individuos que las componen pueden estar en ramas diferentes y a una distancia considerable entre ellas. En general, los marcadores microsatélites empleados permiten identificar una gran variabilidad genética en las accesiones de granadilla estudiadas, la cual se ve reflejada por las diferencias entre las plantas de cada accesión. Además, la poca estructuración geográfica encontrada es el resultado de intercambio de semillas entre productores de 
diferentes departamentos y al tipo de reproducción cruzada presente en la granadilla.

\section{Análisis factorial de correspondencia} múltiple (AFCM)

En el desarrollo del AFCM fueron excluidos los alelos únicos y raros para evitar una mayor dispersión de los individuos que los poseen. Un total de cinco factores fueron identificados por el AFCM con un 39,17\% de inercia total. La Figura 2 muestra la distribución espacial de las accesiones en el plano principal y representa el $18,60 \%$ de inercial total. El análisis revela una fuerte dispersión de las accesiones y menos estructurada que el generado con el árbol de clasificación (neighbor joining). Las pocas accesiones agrupadas que se pueden identificar se entre mezclan con otros departamentos, como es el caso de Tolima (TolLig), Antioquia (AntLig), Boyacá (BoyLig) y Valle del Cauca (ValLig). Las 41 accesiones de granadilla evaluadas se ubicaron de una forma dispersa en el plano principal sin estructuración o agrupación evidente entre los individuos de acuerdo al origen geográfico. Estos resultados, dificultan la identificación de grupos de genotipos diferenciados y al igual que la clasificación arbórea la distancia entre los individuos de una misma accesión es superior que entre ellas.

\section{DISCUSIÓN}

Un set de cinco de diez marcadores microsatélites aislados y caracterizados en maracuyá por Oliveira et al. (2005) presentaron polimorfismo en las 41 accesiones de granadilla evaluadas (Tabla 2). La efectividad de los marcadores alcanzó el 50\% de transferibilidad en la granadilla y el número de alelos observados fue superior (66) a los reportados en maracuyá (35). Por el contrario, en Gulupa la transferibilidad de estos microsatélites fue del $47 \%$, pero los alelos observados fueron monomórficos y no mostraron polimorfismo en los 60 individuos evaluados (ORTIZ et al., 2011). Las diferencias entre estos resultados, puede explicarse debido a que la granadilla presenta un grado de domesticación más incipiente que el maracuyá y además la mayoría de los cultivos en Colombia han sido establecidos con semillas provenientes de plantas silvestre en las zonas donde se iniciaron los sistemas productivos como en Antioquia (Urrao) y el Quindío (Génova). Lo anterior sugiere que la granadilla cultivada presente un síndrome de domesticación parcial, tal como ocurre en muchos frutales andinos (eg. mora, Rubus glaucus Benth; uchuva Physalis peruviana L; y tomate de árbol, Solanum betaceum Cav.), ya que la mayoría de éstos pasaron de ser silvestres a cultivados en unos pocos años (GEPTS, 2002).

La riqueza, frecuencia y el contenido de información polimórfica (PIC) de la mayoría de los alelos confirman que los marcadores evaluados son altamente informativos de acuerdo a los criterios de Botstein et al. (1980), el cual considera que debe tener valores superiores a 0,50. Además, el aumento de PIC está relacionado con la distribución y equilibrio de las frecuencias alélicas en la población (MISSIO et al., 2010), lo cual permite afirmar que los marcadores seleccionados son confiables y efectivos para detectar diversidad genética en genotipos de granadilla.

Los valores de heterocigosidad promedio observada $(H o=0,98)$ y esperada $(H e=0,96)$ muestran que el germoplasma evaluado de granadilla posee una elevada riqueza genética (Tabla 2). Además, la presencia de 7 alelos únicos y de una serie de 13 alelos con baja frecuencia $(\leq 0,060)$, pueden considerarse como marcadores interesantes para la selección, caracterización y conservación de genotipos diferenciados de granadilla. Por otro lado, el alto grado de alogamia asociado a la heterocigosis podría ser un mecanismo de supervivencia y adaptabilidad a condiciones ambientales cambiantes en los entornos naturales donde la especie es cultivada (LOBO, 2006), como el cambio climático que está afectando la agricultura mundial (VERMEULEN et al., 2012). Adicionalmente, esta variabilidad genética observada se apoya en la plasticidad fenotípica de la especie, ya que tiene la capacidad de producir fenotipos diferentes para desarrollarse en nichos ecológicos específicos, como una respuesta de estabilidad en la interacción genotipo x ambiente.

\section{Estructura de la diversidad}

El valor promedio de distancia genética total en la población fue de 0,735 y confirma una alta diversidad genética en las 41 accesiones de granadilla evaluadas con poca estructura (Tabla 3 ). La clasificación arbórea (neighbor joining) muestra tres grandes grupos (Figura 1) con los valores de bootstrap bajos, indicando que la estructuración de los grupos detectados no están consolidados y pueden variar (EFRON, 1979). Por el contrario, las ramas internas están bien soportadas (bootstrap $\geq 50$ ) y ratifican las agrupaciones entre algunas accesiones de un mismo departamento (eg. ValLig, TolLig y PutLig). Las relaciones entre los individuos en su mayoría es leve y no sigue un patrón geográfico de acuerdo al origen de cada accesión, lo cual se debe a que la distribución de los alelos no es sistemática jerarquizada, a excepción de las accesiones que 
presentan alelos únicos y raros. Esta inestabilidad, en la mayoría de los casos ocurre cuando algunos individuos en el momento de la agrupación presentan valores de similaridad intermedia respecto a otros grupos, de modo que no son asignados a uno específico y pueden pertenecer a varias ramas conformada (LAURENTIN, 2009).

El análisis factorial de correspondencia múltiple (AFCM) presentó resultados similares a los arrojados por la clasificación arbórea, pero en el primero las accesiones se localizan más dispersas y no se evidenció un claro agrupamiento por regiones, con excepción del departamento de Putumayo y algunas del Valle del Cauca (Figura 2). El conjunto de resultados de ambos análisis evidencian que las accesiones analizadas son muy heterogéneas genéticamente e indican que hay poca correspondencia con la distribución geográfica de las accesiones. En contraste, Ortiz et al., (2012) reportaron una alta homogeneidad genética en materiales cultivados de gulupa ( $P$. edulis f. edulis) en Colombia con marcadores AFLP, y sin una concordancia con la procedencia de las muestras analizadas por departamento o localidad.

En relación a lo anterior, las accesiones de granadilla cultivadas en Colombia no han sido sometidas a un plan adecuado de selección, ya que los agricultores inconscientemente escogen los mejores frutos en cada cosecha sin tener en cuenta la polinización cruzada. Este tipo de selección fenotípica, solo permite conocer los atributos de los plantas madres, puesto que las semillas de los frutos cosechados constituyen familias de hermanos medios. En consecuencia, los cultivos presentan una degeneración genética de la diversidad en términos de estructuración y de una mayor susceptibilidad a problemas fitosanitarios, como la Mosca del Ovario (Dasiops sp.), la Secadera (Fusarium sp.) y la Virosis (Virus del mosaico de la soya -SMV) que cada vez están afectando más los cultivos e imponen tratamientos químicos para su protección.

La variabilidad genética encontrada en este estudio puede explicarse por la diferenciación geográfica de las accesiones de granadilla evaluadas, el flujo de polen entre diferentes genotipos de poblaciones cercanas y por la introducción de materiales de otras localidades por parte de los agricultores. Esto concuerda con los resultados en otros frutales andinos por Morillo et al. (2005) en plantas de mora (Rubus glaucus), y lo reportado por Bonilla et al. (2008) en uchuva (Physalis peruviana), quienes argumentan que el polimorfismo genético puede estar asociado con la naturaleza alógama de la especie, la cual tiende a favorecer la conservación de un alto porcentaje de genotipos heterocigotos. Los resultados anteriores indican que los marcadores microsatélites son una herramienta poderosa para detectar variabilidad genética respecto a los demás marcadores de ADN estudiados en granadilla.

\section{Implicaciones en el mejoramiento genético}

Este estudio establece una alta variabilidad intraespecífica en los materiales cultivados de granadilla en Colombia. La información generada en esta investigación es de fundamental importancia para los fitomejoradores, ya que establece la estructura genética y diversidad en la granadilla cultivada como base para la búsqueda de genotipos más productivos y resistentes a problemas fitosanitarios (ZHOU et al., 2002). Para desarrollar una estrategia de mejoramiento genético en la granadilla es necesario complementar la información de los marcadores moleculares con datos agromorfológicos de cada accesión, que permitan establecer la verdadera relación entre las variantes fenotípicas y genotípicas. Además, las accesiones con ciertas características de calidad del fruto son fuente de clones y deben ser propuestas como base para la selección de genotipos superiores en la obtención de cultivares más precoces y productivos (LOBO, 2006), partiendo de procesos selectivos en poblaciones locales, con un enfoque en la participación directa de los agricultores. El punto de partida en la estrategia de mejoramiento en la granadilla son las accesiones CunLig01, CunLig03, CunLig05, HuiLig07, QuiLig02, RisLig04 y TolLig02 (Tabla 1), ya que estas cumplen con los parámetros fisicoquímicos requeridos por el mercado, como el peso fruto ( $\geq 120 \mathrm{~g}$ ), el porcentaje de pulpa ( $\geq 50 \%$ ) y los ${ }^{\circ}$ Brix $(\geq 14,5)$. Sin embargo, estas accesiones deben ser evaluadas en campo y realizar ciclos de selección para fijar los caracteres de interés por medio de polinizaciones controladas para evitar el flujo de genes con genotipos no deseados. Adicionalmente, es importante incluir aquellos individuos que posean alelos únicos y raros para enriquecer el acervo genético primario de las accesiones cultivadas como una estrategia para la conservación y mejoramiento de los recursos genéticos en la especie. Lo anterior permitirá la selección asistida por marcadores (SAM) de parentales con amplia distancia genética cuantitativa para el desarrollo de híbridos per se con una alta heterosis en la generación $\mathrm{F}_{1}$. Finalmente, este es el primer estudio de diversidad genética en granadilla con el uso de marcadores microsatélites y propone esta técnica como una herramienta eficaz en la caracterización de germoplasma de la especie, la cual debe ser complementada con estudios agromorfológicos que permitan establecer el grado variabilidad total. 
TABLA 1- Lista de accesiones de granadilla evaluadas en el estudio con el uso de marcadores microsatélites. Características del fruto (PF: peso del fruto (g); \%Pulpa: porcentaje de pulpa; ${ }^{\circ}$ Brix: Solidos solubles totales).

\begin{tabular}{|c|c|c|c|c|}
\hline $\mathbf{N}^{0}$. & Accesión & Departamento & Municipio & PF / \%Pulpa / ${ }^{\circ}$ Brix \\
\hline 1 & AntLig01 & Antioquia & Jardín & $107,0 / 49,1 / 15,8$ \\
\hline 2 & AntLig02 & Antioquia & Urrao & $113,7 / 51,0 / 15,1$ \\
\hline 3 & AntLig03 & Antioquia & Urrao & $155,8 / 52,3 / 14,1$ \\
\hline 4 & BoyLig01 & Boyacá & Buenavista & $133,7 / 42,8 / 15,3$ \\
\hline 5 & BoyLig02 & Boyacá & Buenavista & $115,3 / 50,6 / 16,0$ \\
\hline 6 & BoyLig03 & Boyacá & Buenavista & $118,1 / 52,7 / 16,2$ \\
\hline 7 & BoyLig04 & Boyacá & Tibaná & $83,8 / 30,2 / 15,0$ \\
\hline 8 & BoyLig05 & Boyacá & Somondoco & 80,0 / 44,7 / 17,1 \\
\hline 9 & CalLig01 & Caldas & Aranzazu & $94,9 / 54,1 / 15,0$ \\
\hline 10 & CalLig02 & Caldas & Salamina & $93,1 / 50,4 / 16,3$ \\
\hline 11 & CalLig03 & Caldas & Salamina & $125,8 / 47,2 / 15,7$ \\
\hline 12 & CalLig04 & Caldas & Aranzazu & $113,6 / 52,6 / 16,0$ \\
\hline 13 & CalLig05 & Caldas & Anserma & $125,5 / 48,9 / 15,4$ \\
\hline 14 & CalLig06 & Caldas & Anserma & $105,6 / 55,2 / 16,3$ \\
\hline 15 & CalLig07 & Caldas & Neira & $90,0 / 54,2$ / 15,3 \\
\hline 16 & CunLig01 & Cundinamarca & Venecia & $134,3 / 50,9 / 15,8$ \\
\hline 17 & CunLig02 & Cundinamarca & Arbeláez & $118,4 / 61,0 / 14,5$ \\
\hline 18 & CunLig03 & Cundinamarca & Fusagasuga & $119,6 / 53,7 / 16,3$ \\
\hline 19 & CunLig04 & Cundinamarca & Pasca & $117,1 / 49,1 / 15,2$ \\
\hline 20 & CunLig05 & Cundinamarca & Silvania & $128,4 / 50,2 / 16,1$ \\
\hline 21 & CunLig06 & Cundinamarca & Choachí & $113,2 / 53,9 / 16,1$ \\
\hline 22 & HuiLig03 & Huila & La Argentina & 147,2 / 50,6 / 13,9 \\
\hline 23 & HuiLig06 & Huila & Pitalito & $114,5 / 51,1 / 15,1$ \\
\hline 24 & HuiLig07 & Huila & Garzón & $132,0 / 49,1 / 15,0$ \\
\hline 25 & PutLig01 & Putumayo & Sibundoy & $76,8 / 47,3 / 15,3$ \\
\hline 26 & QuiLig01 & Quindío & Génova & $118,3 / 50,9 / 15,8$ \\
\hline 27 & QuiLig02 & Quindío & Génova & $124,4 / 50,1 / 14,7$ \\
\hline 28 & QuiLig05 & Quindío & Salento & 79,2 / 37,5 / 15,2 \\
\hline 29 & RisLig02 & Risaralda & Santa Rosa & $131,4 / 46,2$ / 14,3 \\
\hline 30 & RisLig03 & Risaralda & Apia & $140,0 / 42,2 / 16,2$ \\
\hline 31 & RisLig04 & Risaralda & Apia & $134,1 / 49,0 / 15,3$ \\
\hline 32 & TolLig01 & Tolima & Cajamarca & $116,4 / 48,0 / 17,1$ \\
\hline 33 & TolLig02 & Tolima & Cajamarca & $137,4 / 49,0 / 15,7$ \\
\hline 34 & TolLig03 & Tolima & Ibagué & $97,8 / 54,5 / 14,7$ \\
\hline 35 & ValLig01 & Valle del Cauca & Yotoco & $96,0 / 44,0 / 15,8$ \\
\hline 36 & ValLig02 & Valle del Cauca & Restrepo & $102,2 / 53,0 / 15,2$ \\
\hline 37 & ValLig03 & Valle del Cauca & Tuluá & $109,3 / 47,1 / 14,6$ \\
\hline 38 & ValLig04 & Valle del Cauca & Ginebra & $171,2 / 50,9 / 15,7$ \\
\hline 39 & ValLig05 & Valle del Cauca & Versalles & $118,6 / 45,7 / 15,0$ \\
\hline 40 & ValLig06 & Valle del Cauca & Versalles & $103,8 / 45,3 / 14,9$ \\
\hline 41 & ValLig07 & Valle del Cauca & El Cerrito & $96,4 / 49,0 / 15,9$ \\
\hline
\end{tabular}




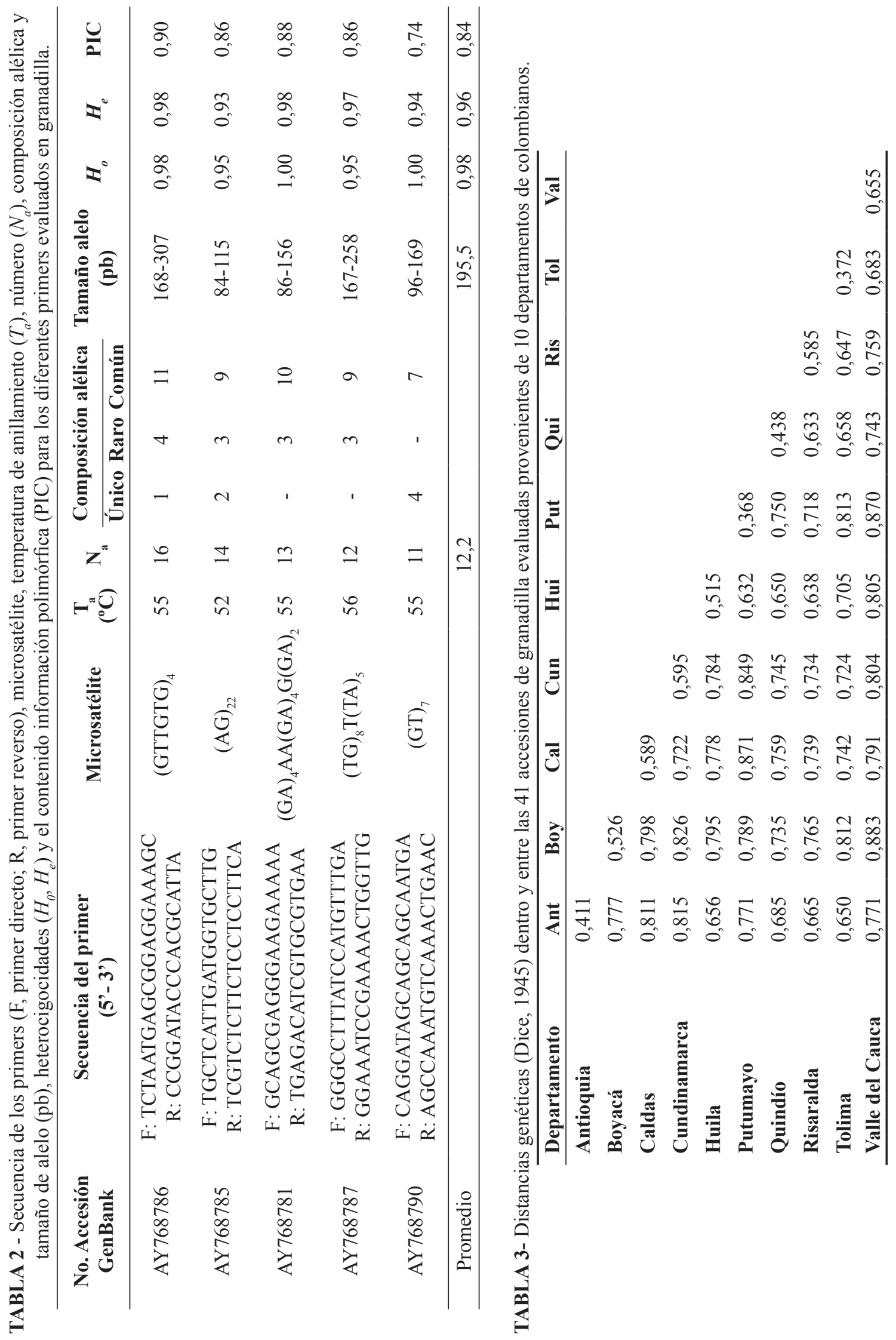




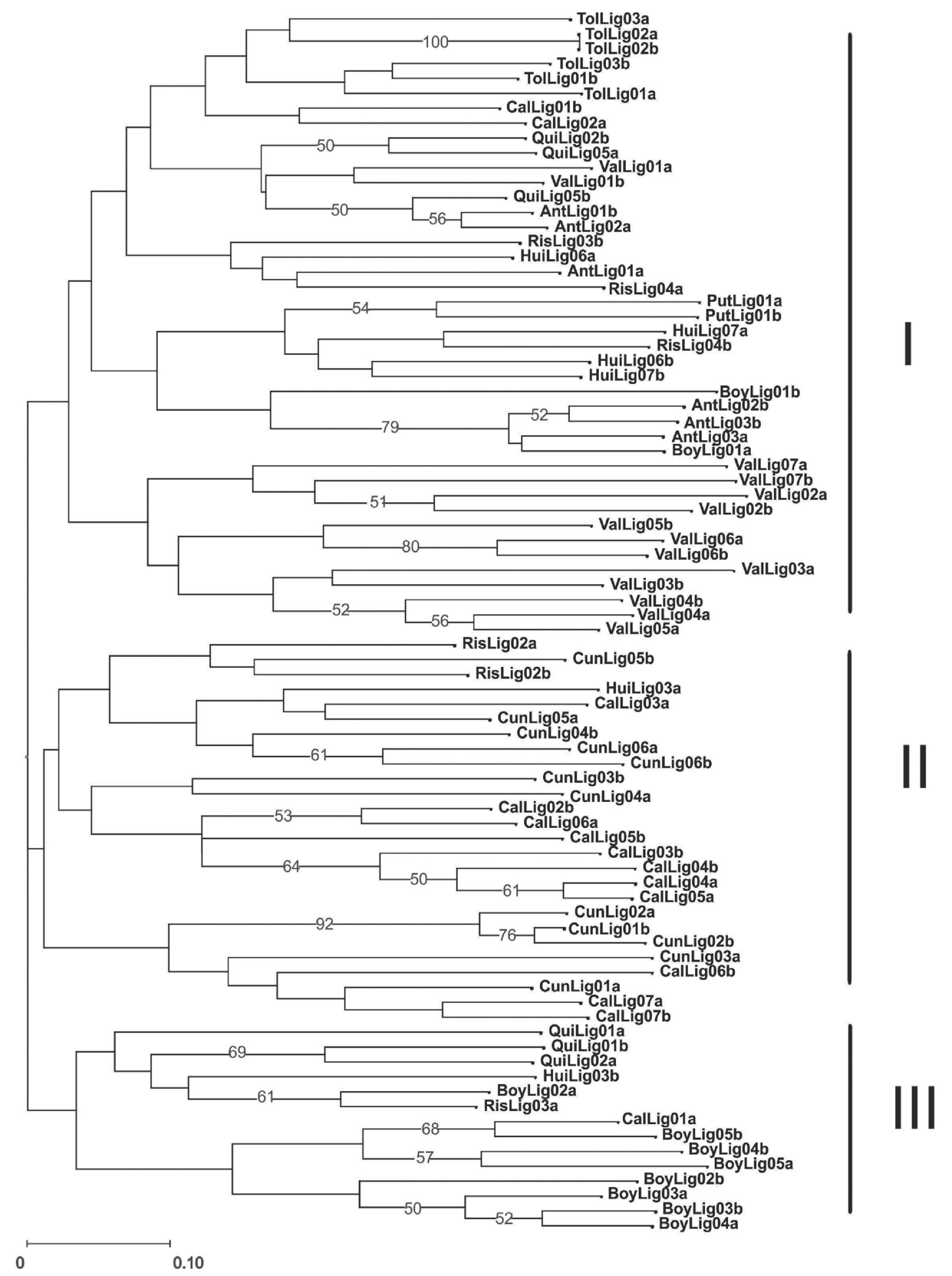

FIGURA 1- Análisis de clasificación arbórea neighbor joining (distancias de Sokal \& Michener) de 41 accesiones de granadilla provenientes de 10 departamentos de Colombia. Solo los valores de bootstrap $\geq 50 \%$ son mostrados. 


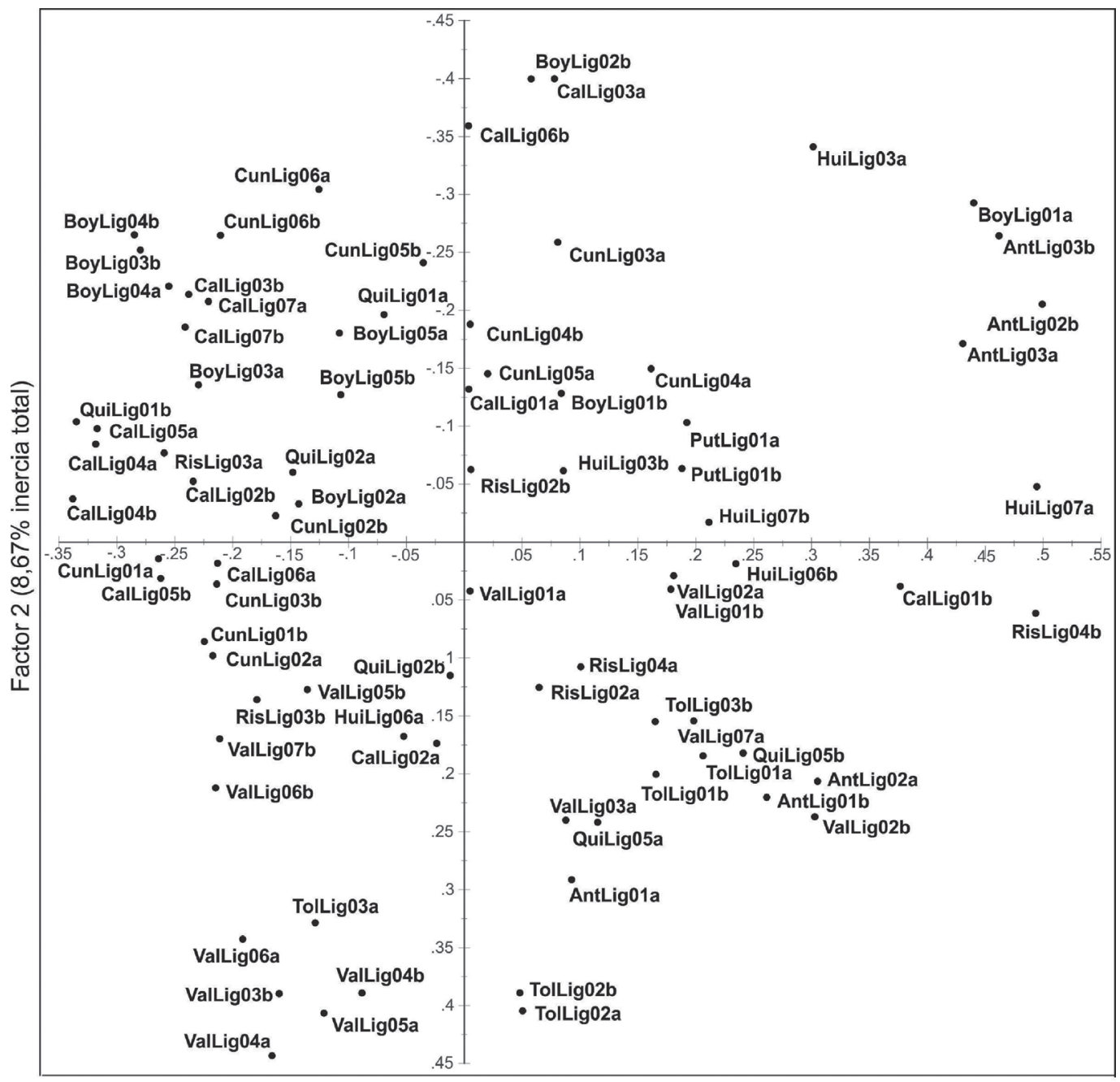

Factor $1(9,93 \%$ inercia total)

FIGURA 2- Distribución espacial de las 41 accesiones de granadilla evaluadas en el plano principal del análisis factorial de correspondencia múltiple (AFCM).

\section{CONCLUSIONES}

La caracterización molecular con el uso de marcadores microsatélites permitió establecer que el germoplasma de granadilla evaluado en esta investigación presentó un alto grado de variabilidad genética $(0,735)$. Sin embargo, la distribución de los individuos en los análisis de clasificación arbórea y factorial no siguen un parámetro geográfico definido en la mayoría de las accesiones.

El conjunto de resultados sugieren que los procesos de selección de los materiales de siembra, la polinización cruzada presente, el intercambio de semillas entre productores y una domesticación incipiente han contribuido con la escaza estructuración genética de las accesiones evaluadas, reflejada en la variabilidad intraespecífica detectada en los materiales cultivados de granadilla en Colombia.

\section{AGRADECIMIENTOS}

Los autores agradecen el Ministerio de Agricultura y Desarrollo Rural de la Republica de Colombia (MADR) por el financiamiento de proyecto "Aprovechamiento de la diversidad del maracuyá amarillo ( $P$. edulis f. flavicarpa Degener), la gulupa (P. edulis f. edulis Sims) y la granadilla (P. ligularis Juss.) para mejorar y diversificar los sistemas de producción en Colombia 074-2008L6772-3447". A la dirección de Investigación, Creatividad e Innovación de la Universidad Jorge Tadeo Lozano por el apoyo en el desarrollo del Proyecto. 


\section{REFERENCIAS}

BILLOTTE, N.; LAGODA, P.J.L.; RISTERUCCI, A.M.; BAURENS, C. Microsatellite-enriched libraries: applied methodology for the development of SSR markers in tropical crops. Fruits, Paris, v. 54, p.277- 288, 1999.

BONILLA, M.L.; ESPINOSA, K.; POSSO, A.M.; VÁSQUEZ, HD.; MUÑOZ, J.E. Caracterización molecular de 43 accesiones de uchuva de seis departamentos de Colombia. Acta Agronómica, Palmira, v.57, n.2, p.109-115, 2008.

BOTSTEIN, D.; WHITE, L.; SKOLMICK, H.; DAVIS, W. Construction of a genetic linkage map in man using restriction fragment length polymorphism. American Journal of Human Genetics, Chicago, v.32, p.314-331, 1980.

CROCHEMORE, M.; CORREA, H.; ESTEVEZ, L. Genetic Diversity in Passion Fruit (Passiflora spp.) evaluated by RAPD markers. Brazilian Archives of Biology and Technology, Curitiba, v.46, n.4, p. 521-527, 2003.

DICE, L. Measures of the amount of ecological association beetwen species. Ecology, Durdham, v.26, p.297-302, 1945

DOYLE, J.J.; DOYLE, J.L. Isolation of plant DNA from fresh tissue. Focus, Rockville, v.1, p.13-15. 1991.

EFRON, B. Bootstrap methods: another look at the jackknife. Annals Statistics, Hayward, v.7, n. p.126, 1979.

FAJARDO, D.; ANGEL F.; GRUM, M.; TOHME, J.; LOBO, M.; ROCA, W.; SÁNCHEZ, I. Genetic variation analysis of the genus Passiflora L. using RAPD markers. Euphytica, Wagenigen, v.101, p.341-347, 1998.

FRANCO, Y.; ALZATE, F.; PELÁEZ, J.M. Factores ambientales incidentes en la población de Xylocopa y su efecto en el cultivo de granadilla en tres veredas del municipio de Guarne (Colombia). Revista Universidad Católica de Oriente, Medellín, n.24, p.73-88, 2007.
GEPTS, P. A comparison between crop domestication, classical plant breeding and genetic engineering. Crop Science, Madison, v.42, p.1780-1790, 2002

GONÇALVES-VIDIGAL, M.C.; BENCHIMOL, L. Development and application of microsatellites in plant breeding. Crop Breeding and Applied Biotechnology, Londrina, v.11, p.66-72, 2011.

KALIA, R.K.; RAI, M.K.; KALIA, S.; SINGH, R.; DHAWAN, A.K. Microsatellite markers: an overview of the recent progress in plants. Euphytica, Wageningen, v.177, p.309-334, 2011.

LAURENTIN, H. Data analysis for molecular characterization of plant genetic resources. Genetic Resources and Crop Evolution, Dordrecht, v.56, p.277-292, 2009.

LOBO, M. Recursos genéticos y mejoramiento de frutales andinos: una visión conceptual. Revista Corpoica - Ciencia y Tecnología Agropecuaria, Bogotá, v.7, n.2, p.40-54, 2006

MISSIO, R.F.; TEIXERA, E.; ZAMBOLIM, E.M.; ZAMBOLIM, L.; CRUZ, C.M.; SUSSUMU, N. Polymorphic information content of SSR markers for Coffea spp. Crop Breeding and Applied Biotechnology, Londrina, v.10, p.89-94, 2010.

MORILLO, A.; MORILLO, Y.; MUÑOZ, J.E.; VÁSQUEZ, H.D.; ZAMORANO, A. Caracterización molecular con microsatélites aleatorios RAMs de la colección de mora, Rubus spp. de la Universidad Nacional de Colombia sede Palmira. Acta Agronómica, Palmira, v.54, n.2, p.15-24, 2005.

OCAMPO, J.; COPPENS D’EECKENBRUGGE, G.; OLANO, C.; SCHNELL, R. AFLP analysis for the study of genetic relationships among cultivated Passiflora species of the subgenera Passiflora and Tacsonia. Proceedings of the Interamerican Society for Tropical Horticulture, Miami, v.47, p.72-76, 2004.

OCAMPO, J.A.; COPPENS D'EECKENBRUGGE, G.; RESTREPO, M.; SALAZAR, M.; JARVIS, A. Diversity of Colombian Passifloraceae: biogeography and an updated list for conservation. Biota Colombiana, Colombia, v.8, n.1, p.1-45, 2007a. 
OCAMPO, J.; COPPENS D’EECKENBRUGGE, G.; RISTERUCCI，A-M.; DAMBIER，D.; OLLITRAULT, P. Papaya genetic diversity assessed with microsatellite markers in germplasm from the Caribbean region. Acta Horticulturae, The Hague, v.740, p.93-102, 2007 b.

OCAMPO, J.; URREA, R.; WYCKHUYS, K.; SALAZAR, M. Aprovechamiento de la variabilidad genética del maracuyá (Passiflora edulis f. flavicarpa Degener) como base para un programa de fitomejoramiento en Colombia. Acta Agronómica, Palmira, v.62, n.4, p.352-360, 2013.

OLIVEIRA, J.; PÁDUA, G.; ZUCCHI, I.; CAMARGO, A.; FUNGARO, M.H.P.; VIEIRA M.L. C. Development and characterization of microsatellite markers from the yellow passion fruit (Passiflora edulis f. flavicarpa). Molecular Ecology Notes, Oxford, v.5, p.331-333, 2005.

ORTIZ, D.; BOHÓRQUEZ, A.; DUQUE, M.C.; TOHME, J.; CUELLAR, D.; MOSQUERA, T. Evaluating purple passion fruit (Passiflora edulis Sims f. edulis) genetic variability in individuals from commercial plantations in Colombia. Genetic Resources and Crop Evolution, Dordrecht, v.59, n.6, p.1089-1099, 2012.

PADUA, J.G.; OLIVEIRA, E.J.; ZUCCHI, M.I.; OLIVEIRA, G.C.X.; CAMARGO, LEA.; VIEIRA, M.L.C. Isolation and characterization of microsatellite markers from the sweet passion fruit (Passiflora alata Curtis: Passifloraceae). Molecular Ecology Notes, Oxford, v.5, p.863-865, 2005.

SAITOU, N.; NEI, M. 1987. The neighborjoining method: a new method for reconstructing phylogenetic trees. Molecular Biology and Evolution,Chicago, v.44, p.406-425, 1987.

SANCHEZ, I.; FAJARDO, A.; GRUM, M.; DUQUE, M.; LOBO, M.; TOHME, J.; ROCA, W. Variability of chloroplast DNA in the genus Passiflora L. Euphytica, Wageningen, v.106, p.15-26, 1999.

SANTOS, L.F.; OLIVEIRA, E.J.; SANTOS, A.; MORAES, F.; LELES, J.; PADUA, J. ISSR markers as a tool for the assessment of genetic diversity in Passiflora. Biochemical Genetic, Dordrecht, v.49, p.540-554, 2011.
SEGURA, S.; COPPENS D’EECKENBRUGGE, G.; BOHÓRQUEZ, A.; OLLITRAULT, P.; TOHMÉ, J. An AFLP study of the genus Passiflora focusing on subgenus Tacsonia. Genetic Resources and Crop Evolution, Dordrecht, v.9, p.111-123, 2002.

SEMAGN, K.; BJØRNSTAD, A.; NDJIONDJOP, M.N. An overview of molecular marker methods for plants. African Journal of Biotechnology, Nigeria, v.5, n.25, p.2540-2568, 2006.

SNOW, N.; MACDOUGAL, J.M. New chromosome reports in Passiflora (Passifloraceae). Systematic Botany, Kent, v.18, n.2, p.261-273, 1993.

VERMEULEN, S.; ZOUGMORE, R.; WOLLENBERG, E.; THORNTON, P.; NELSON, G.; KRISTJANSON, P.; KINYANGI4, J.; JARVIS, A.; HANSEN, J.; CHALLINOR, C.; CAMPBELL, B.; AGGARWAL, P. Climate change, agriculture and food security: a global partnership to link research and action for low-income agricultural producers and consumers. Current Opinion in Environmental Sustainability, Bloomington v.4, n.1, p.128-133, 2012.

YOCKTENG, R.; NADOT, S. Phylogenetic relationships among Passiflora species based on the glutamine synthetase nuclear gene expressed in chloroplast (ncpGS). Molecular Phylogenetics and Evolution, Orlando, v.31, p.379-396, 2004.

ZHOU, X.; CARTER, T.E.; CUÍ, Z.; MIYAZAKI, S.; BURTON, J. Genetic diversity patterns in Japanese soybean cultivars based on coefficient of parentage. Crop Science, Madison, v.42, p.1331-1342, 2002. 